\title{
Correction to: Stepwise Disease Progression Model of Subsolid Lung Adenocarcinoma with Cystic Airspaces
}

\author{
Woohyun Jung, MD ${ }^{1}$, Sukki Cho, MD, PhD ${ }^{1,2}$, Sungwon Yum, BS ${ }^{1}$, Jin-Haeng Chung, MD, PhD ${ }^{3,4}$, \\ Kyung Won Lee, MD, PhD ${ }^{5,6}$, Kwhanmien Kim, MD, $\mathrm{PhD}^{1,2}$, Choon Taek Lee, MD, PhD ${ }^{7,8}$, and \\ Sanghoon Jheon, MD, $\mathbf{P h D}^{1,2}$
}

${ }^{1}$ Department of Thoracic and Cardiovascular Surgery, Seoul National University Bundang Hospital, Seongnam-si, Gyeonggi-do, Republic of Korea; ${ }^{2}$ Department of Thoracic and Cardiovascular Surgery, Seoul National University College of Medicine, Seoul, Republic of Korea; ${ }^{3}$ Department of Pathology, Seoul National University Bundang Hospital, Gyeonggi-do, Republic of Korea; ${ }^{4}$ Department of Pathology, Seoul National University College of Medicine, Seoul, Republic of Korea; ${ }^{5}$ Department of Radiology, Seoul National University Bundang Hospital, Gyeonggi-do, Republic of Korea; ${ }^{6}$ Department of Radiology, Seoul National University College of Medicine, Seoul, Republic of Korea; ${ }^{7}$ Department of Internal Medicine, Seoul National University Bundang Hospital, Gyeonggi-do, Republic of Korea; ${ }^{8}$ Department of Internal Medicine, Seoul National University College of Medicine, Seoul, Republic of Korea

\section{CORRECTION TO:}

ANN SURG ONCOL

HTTPS://DOI.ORG/10.1245/S10434-020-08508-4

In the original article there are errors in Fig. 3. Following is the corrected figure.

The original article can be found online at https://doi.org/10.1245/ s10434-020-08508-4.

(C) Society of Surgical Oncology 2020

Published Online: 8 July 2020

S. Cho, MD, PhD

e-mail: skcho@snubh.org 


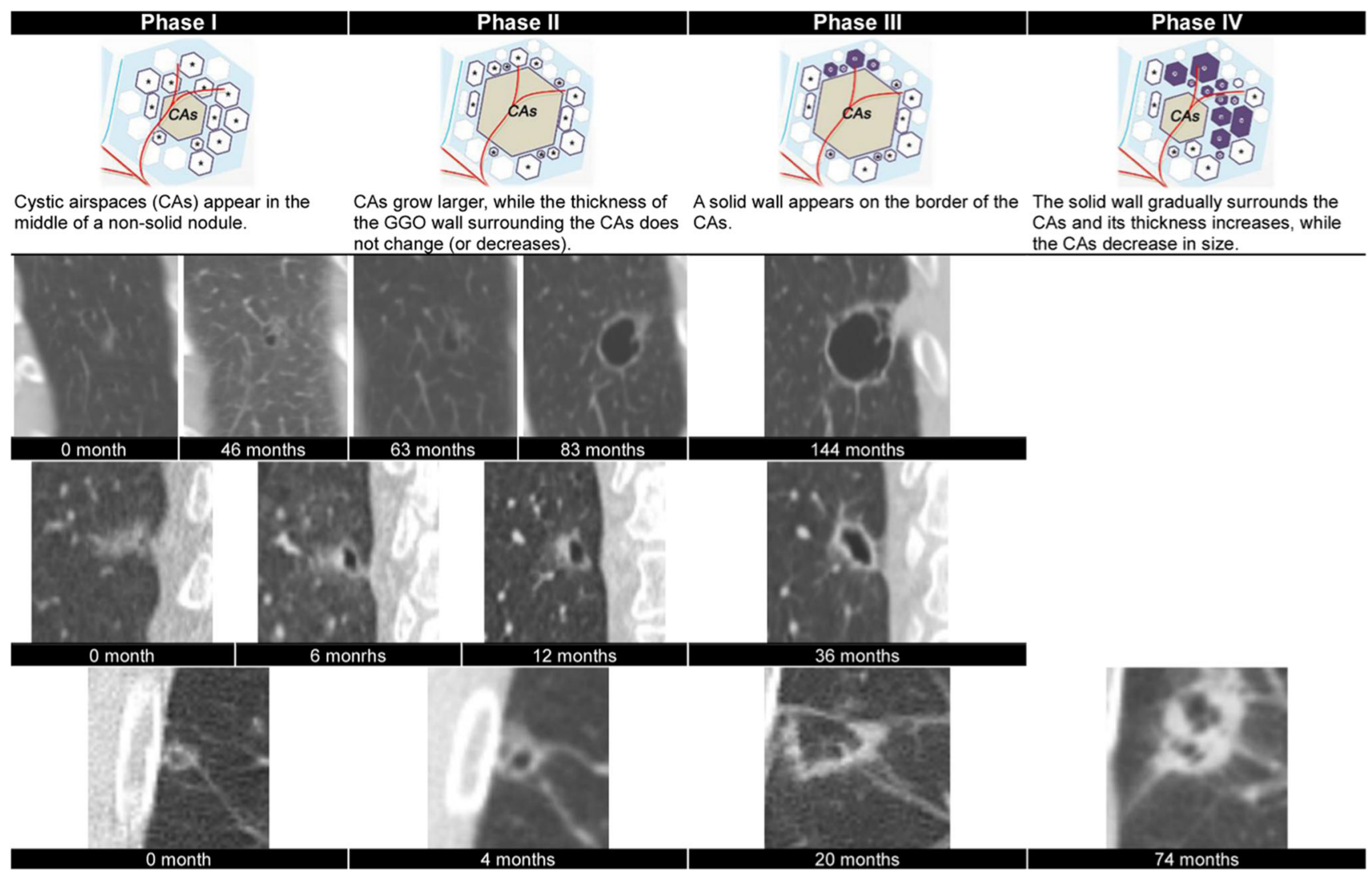

FIG. 3 Radiologic stepwise disease progression model of LACA. LACA lung adenocarcinoma with cystic airspaces, GGO ground-glass opacity, CAs cystic airspaces

Publisher's Note Springer Nature remains neutral with regard to jurisdictional claims in published maps and institutional affiliations. 